\title{
Projectile - Mass asymmetry systematics for low energy incomplete fusion
}

\author{
Pushpendra P. Singh ${ }^{1}$, a , Abhishek Yadav ${ }^{2}$, Vijay R. Sharma ${ }^{3}$, Manoj K. Sharma ${ }^{4}$, Pawan Kumar ${ }^{1}$, Rudra N. Sahoo ${ }^{1}$, \\ R. Kumar' ${ }^{2}$, R. P. Singh², S. Muralithar ${ }^{2}$, B. P. Singh ${ }^{3}$, R. K. Bhowmik ${ }^{2}$, R. Prasad ${ }^{3}$, and the AMU-IUAC collaboration \\ ${ }^{1}$ Department of Physics, Indian Institute of Technology Ropar, Rupnagar, PB - 140 001, India \\ ${ }^{2}$ NP-Division, Inter-University Accelerator Centre, New Delhi - 110 067, India \\ ${ }^{3}$ Acc. Lab., Department of Physics, A. M. University, Aligarh, UP - 202 002, India \\ ${ }^{4}$ Physics Department, S. V. College, Aligarh, UP - 202 001, India
}

\begin{abstract}
In the present work, low energy incomplete fusion (ICF) in which only a part of projectile fuses with target nucleus has been investigated in terms of various entrance channel parameters. The ICF strength function has been extracted from the analysis of experimental excitation functions (EFs) measured for different projectile-target combinations from near- to well above- barrier energies in ${ }^{12} \mathrm{C},{ }^{16} \mathrm{O}$ (from $1.02 \mathrm{~V}_{b}$ to $\left.1.64 \mathrm{~V}_{b}\right)+{ }^{169} \mathrm{Tm}$ systems. Experimental EFs have been analysed in the framework statistical model code PACE4 based on the idea of equilibrated compound nucleus decay. It has been found that the value of ICF fraction $\left(\mathrm{F}_{I C F}\right)$ increases with incident projectile energy. A substantial fraction of ICF $\left(\mathrm{F}_{I C F} \approx 7 \%\right)$ has been accounted even at energy as low as $\approx 7.5 \%$ above the barrier (at relative velocity $v_{\text {rel }} \approx 0.027$ ) in ${ }^{12} \mathrm{C}+{ }^{169} \mathrm{Tm}$ system, and $\mathrm{F}_{I C F} \approx 10 \%$ at $v_{\text {rel }} \approx 0.014$ in ${ }^{16} \mathrm{O}+{ }^{169} \mathrm{Tm}$ system. The probability of ICF is discussed in light of the Morgenstern's mass-asymmetry systematics. The value of $\mathrm{F}_{I C F}$ for ${ }^{16} \mathrm{O}+{ }^{169} \mathrm{Tm}$ systems is found to be $18.3 \%$ higher than that observed for ${ }^{12} \mathrm{C}+{ }^{169} \mathrm{Tm}$ systems. Present results together with the re-analysis of existing data for nearby systems conclusively demonstrate strong competition of ICF with CF even at slightly above barrier energies, and strong projectile dependence that seems to supplement the Morgenstern's systematics.
\end{abstract}

\section{Introduction}

Understanding the dynamics of light-heavy-ion $(\mathrm{A} \geq 20)$ induced low energy incomplete fusion (ICF) gained significant interest in recent years [1-10]. The most dominating mode of reaction from near- to above- barrier energies is complete fusion $(\mathrm{CF})$ in which entire projectile fuses with target nucleus and leads to a completely fused composite system essentially via single route. However, a significant ICF contribution has been observed at slightly above barrier energies $[11,12]$. The existence of ICF at low incident energy has triggered scientific thrust to probe different aspects of underlying dynamics which are not yet fully understood [3, 4, 13-15]. The important findings of previous studies on ICF are; $(i)$ at high $\ell$-values $\left(\ell \geq \ell_{\text {crit }}\right)$, incident projectile breaks up into constituents to provide sustainable input angular momenta, and fuses partly with target nucleus [4, 16-19], (ii) the partially-fused-compositesystem forms with less mass/charge and recoil velocity as that of completely fused composite (CFC)-system [10, 2024], and (iii) the fraction of ICF has been found to be large for more mass-asymmetric systems [28]. However, some of the experimental results likely to contradict previous findings [25-27]. For example, according to Morgenstern's systematics [28], the fraction of ICF is expected to be large for more mass-asymmetric systems at constant

\footnotetext{
a e-mail: pps@iitrpr.ac.in
}

value of relative velocity $\left(v_{r e l}=\sqrt{2(E c m-V b) / \mu} / \mathrm{c}\right)$. In recent reports $[11,12]$, the variation of ICF fraction with $\mu_{A}$ do not found to obey Morgenstern's systematics. While, the data obtained for individual projectiles $\left({ }^{12} \mathrm{C}\right.$ and $\left.{ }^{16} \mathrm{O}\right)$ have been fairly explained by the given systematics. This observation may be considered as a supplement to the Morgenstern's systematics but rather poorly known, therefore, need to be supported by further measurements and continues to be an active area of investigations.

Some of the outstanding issues to be probed are the effects of; ( $i$ ) projectile energy, (ii) mass-asymmetry of interacting partners $\left(\mu_{A}=A_{T} / A_{T+P}\right)$, (iii) input $\ell$-values, and (iv) the shape of projectile and target nuclei on the onset and strength of ICF. In order to understand these issues, several experiments have been performed at the Inter-University Accelerator Centre (IUAC), New Delhi. In the present work, the fraction of ICF has been deduced in ${ }^{12} \mathrm{C}+{ }^{169} \mathrm{Tm}$ system at energies ranging from $1.02 \mathrm{~V}_{b}$ to $1.64 \mathrm{~V}_{b}\left(\mathrm{~V}_{b}=54.94 \mathrm{MeV}\right)$. The EFs of different residues expected to be populated via $\mathrm{CF}$ and/or ICF have been measured. Present data along with the data from the ref.[29] are compared with the previously reported ${ }^{16} \mathrm{O}$ results [11] to display projectile structure effect on ICF dynamics. A comparison of results from nearly systems, ${ }^{12} \mathrm{C}+{ }^{128} \mathrm{Te},{ }^{165} \mathrm{Ho},{ }^{169} \mathrm{Tm}$ and ${ }^{16} \mathrm{O}+{ }^{103} \mathrm{Rh},{ }^{159} \mathrm{~Tb},{ }^{169} \mathrm{Tm}[11$, $12,30,31]$, is presented to describe mass-asymmetry 
(and/or projectile) dependence on the onset and strength of ICF.

\section{Experimental \& Data Reduction Methodology}

Experiments have been carried out at the IUAC New Delhi using activation technique and off-line $\gamma$-spectroscopy, similar to what has been used in refs.[11, 12]. A brief description of experimental details is given here for ready reference. Natural ${ }^{169} \mathrm{Tm}$ (abundance $=100 \%$ ) targets of thickness $\mathrm{t}_{m} \approx 1 \mathrm{mg}-\mathrm{cm}^{-2}$ were prepared by rolling technique, and thickness has been measured using $\alpha$ transmission method. In the present work, the energy spread and beam intensity variations have been avoided by adopting single target irradiation methodology for each projectile energy. Irradiations were carried out using ${ }^{12} \mathrm{C}$ beam $\left(\mathrm{E}_{\text {beam }}=\approx 54-90 \mathrm{MeV}\right.$ with beam current $\approx 20$ $30 \mathrm{nA}$ ) delivered from 15UD Pelletron accelerator in a small specially deigned irradiation chamber. The constant beam current was maintained throughout the irradiations so that the uncertainty in the production cross-sections due to beam current fluctuations may be minimised. In order to detect the residues of small half-lives $\left(t_{1 / 2} \approx 5-10\right.$ minutes), in-situ measurements of $\gamma$-activities were performed off-line using two 100cc active volume HPGe detectors. Relevant portion of a $\gamma$-ray spectra obtained at energy $\approx 89 \mathrm{MeV}$ is shown in Fig.1. Evaporation residues have been identified by their characteristic $\gamma$-lines, and the decay curve analysis.

\subsection{Measurement of production cross-sections}

The projectile energy dependent reaction cross-sections $\left(\sigma_{E R}\right)$, for different radio-nuclides have been determined using the following expression [12];

$$
\sigma_{E R}=\frac{C_{t=0}}{N_{0} \theta \phi G_{\varepsilon} K\left[1-\exp \left(-\lambda t_{1}\right)\right]}
$$

Where:

$C_{t=0}=$ The count rate at zero time, i.e., just after the irradiation,

$N_{0}=$ Initial number of nuclei in the target smaple,

$\theta=$ Branching ratio of the characteristic $\gamma$-rays assigned to different reaction products,

$\phi=$ The incident beam flux,

$G_{\varepsilon}=$ Geometry dependent efficiency of the HPGe detector,

$K=[1-\exp (-\mu d)] / \mu d=$ The self absorption correction factor for the $\gamma$-rays in the material of the sample of thickness $d\left(\mathrm{gm} / \mathrm{cm}^{2}\right)$ and the absorption coefficient $\mu\left(\mathrm{cm}^{2} / \mathrm{gm}\right)$ $\lambda=$ Decay constant of the radio-nuclides, and $t_{1}=$ Time of irradiation.

The errors in the measured production cross-sections of evaporation residues may arise due to various factors, like; $(i)$ non-uniform thickness of the samples, i.e., the inaccurate estimate of foil thickness may lead to the uncertainty in the determination of the number of target nuclei in the sample. However, in order to check the uniformity

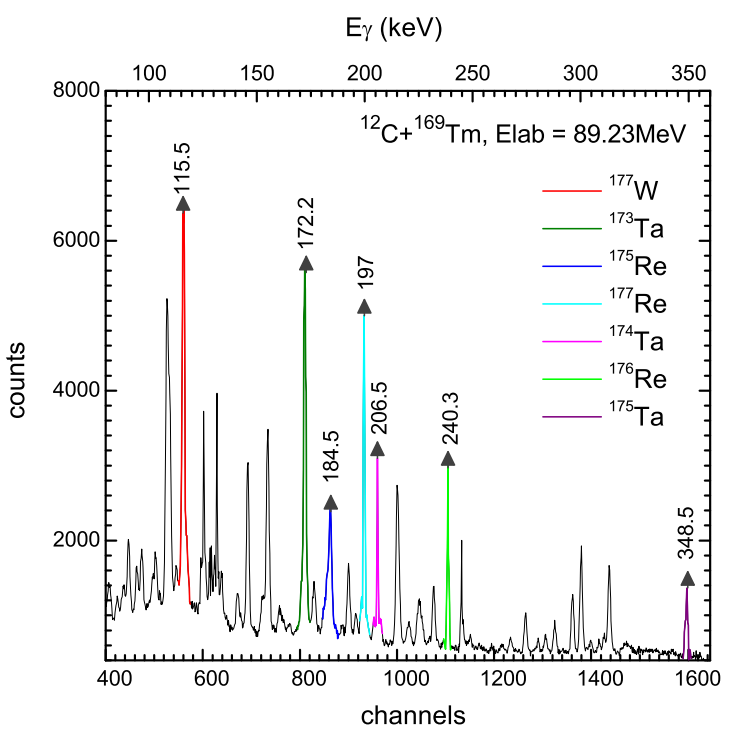

Figure 1. A representative section of $\gamma$-spectra obtained at projectile energy $\mathrm{E}_{\text {lab }} \approx 89.23 \mathrm{MeV}$ in ${ }^{12} \mathrm{C}+{ }^{169} \mathrm{Tm}$ system. Peaks corresponding to different reaction products expected to be populated via CF and/or ICF are marked.

of the sample, thickness of each sample was measured at different positions using $\alpha$-transmission method. The error in the thickness of the sample is estimated to be $\approx 1 \%$. (ii) Fluctuations in the beam current may result in the variation of incident flux, as such proper care has been taken to maintain the beam current constant so that the error due to beam current fluctuations may be minimised. (iii) Uncertainty in the determination of geometry dependent spectrometer efficiency. The error in the efficiency determination due to the statistical fluctuations in counts is estimated to be less than $2 \%$. (iv) The loss of product nuclei recoiling out of the target may introduce large errors in the measured cross-sections. The thickness of the catcher foils used in the present work were sufficient to stop even the most energetic residues. However, in the present measurements both the sample and the catcher foils were counted together and hence, the losses due to the recoiling of nuclei is avoided. (v) Dead time of the spectrometer was kept less than $10 \%$ by suitably adjusting sample-detector distance. The overall error in $\sigma_{E R}$ is estimated to be $\approx 13 \%$.

\subsection{Analysis of EFs with PACE4}

Theoretical production cross-sections of evaporation residues populated via $\mathrm{CF}$ channels have been obtained using code PACE4 [32]. The code PACE4 is based on Hauser-Feshbach approach. It may be pointed out that the ICF and PE-emission are not taken into consideration in this code. The cross-sections for evaporation residues are calculated using Bass formula [33]. The de-excitation of the compound nucleus is followed by Monte-Carlo procedure. The projections of angular momentum are calculated at each stage of de-excitation, which enables the 

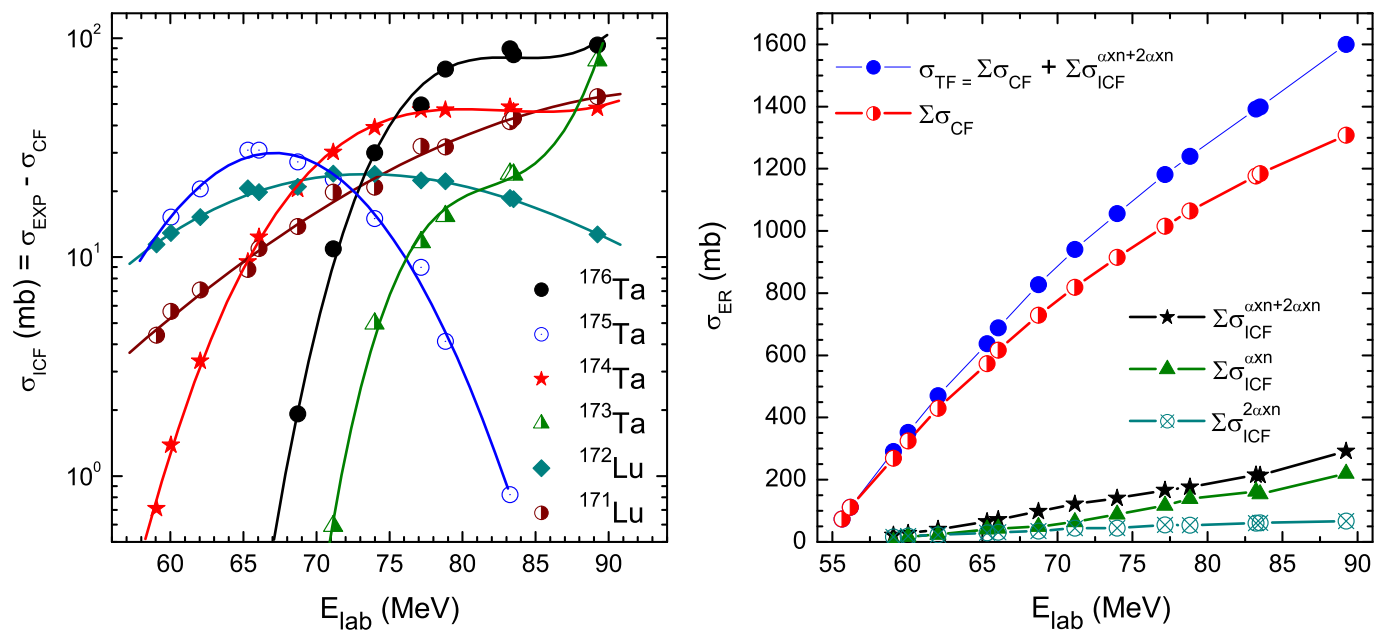

Figure 2. (left) Experimentally measured and systematically deduced (see text for deduction procedure) EFs of ICF residues, (right) Total fusion cross-section $\left(\sigma_{T F}=\Sigma \sigma_{C F}+\Sigma \sigma_{I C F}\right)$ along with the sum of all CF-channels $\left(\Sigma \sigma_{C F}\right)$ and ICF-channels $\left(\Sigma \sigma_{I C F}\right)$ as a function of incident projectile energy. Solid curves represent best fit to the data points.

determination of angular distribution of the emitted particles. The optical model parameters for neutrons, protons and $\alpha$-particles are used as default in the code. The $\gamma$-ray strength functions for $E_{1}, E_{2}$ and $M_{1}$ transition were taken from tables of the literature [34].

The partial cross-section $\left(\sigma_{\ell}\right)$ for the formation of $\mathrm{CN}$ at angular momentum $\ell$ and specific bombarding energy $\mathrm{E}$, is given by,

$$
\sigma_{\ell}=\frac{\lambda^{2}}{4 \pi}(2 \ell+1) T_{\ell}
$$

here, $\lambda$ is the reduced wavelength. The transmission coefficients $T_{\ell}$ may be given by the expression,

$$
T_{\ell}=\left[1+\exp \left(\frac{\ell-\ell_{\max }}{\Delta}\right)\right]^{-1}
$$

Where, $\Delta$ is the diffuseness parameter and $\ell_{\max }$ the maximum value of $\ell$ detained by total fusion cross-section,

$$
\sigma_{F}=\sum_{\ell=0}^{\infty} \sigma_{\ell}
$$

The transmission coefficients for the evaporation of light particles (n, $\mathrm{p}$ and $\alpha$ ) during the de-excitation are obtained by optical model calculations. The level density in this code is calculated using the expression $a=A / K$, where; $\mathrm{A}$ is the atomic mass number and $K$ is a parameter called level density parameter. In these calculations $K$ is an important parameter and affects the equilibrium component [35]. As such, in order to show the effect of variation of $K$ on calculated EFs, different values of $K(8,9$ and 10) have been tested. It may be pointed out that, it might be possible to predict all the EFs with different values of parameters of the code for individual channels. However, it is not reasonable from the physics point of view. In the present work, a value of $K=8$ is found to give a satisfactory reproduction of experimental data for $\mathrm{CF}$-channels within the experimental uncertainties.

\subsection{Estimation of ICF fraction}

Experimentally measured EFs of ${ }^{175,176,177,178} \mathrm{Re},{ }^{177} \mathrm{~W}$, ${ }^{173,174,175,176} \mathrm{Ta}$, and ${ }^{171,172} \mathrm{Lu}$ residues have been analysed using PACE4 to examine the extent to which the observed EFs can be described in terms of equilibrated compound nucleus $(\mathrm{CN})$ decay. Since this code does not take ICF into account, therefore, any deviation in the experimental EFs from the theoretical ones may be attributed to the onset of ICF. It has already been pointed out that the level density parameter $(\mathrm{a}=\mathrm{A} / \mathrm{K})$ is an important input parameter of this code. $\mathrm{A}$ value of $\mathrm{a}=\mathrm{A} / 8 \mathrm{MeV}^{-1}$ has been chosen because it reproduced the ratio of two dominant channels $\sigma 4 \mathrm{n}\left({ }^{177} \mathrm{Re}\right) / \sigma 5 \mathrm{n}\left({ }^{176} \mathrm{Re}\right)$. Therefore, the value of $\mathrm{a}=\mathrm{A} / 8 \mathrm{MeV}^{-1}$ can be used as default parameter for further analysis within the tested energy range. PACE4 confirmed the production of ${ }^{177,176} \mathrm{Re}$ residues through equilibrated $\mathrm{CN}$-decay via emission of $4 / 5$ neutrons from excited ${ }^{181} \mathrm{Re}^{\star}$ formed via $\mathrm{CF}$, and ${ }^{175} \mathrm{Re}(6 \mathrm{n})$ and ${ }^{177} \mathrm{~W}(\mathrm{p} 3 \mathrm{n})$ via corresponding CF-channels. While, the EFs of ${ }^{173,174,175,176} \mathrm{Ta}(\alpha \mathrm{xn})$ residues have been found to be significantly enhanced than that predicted by PACE4. The enhancement in $\alpha$-emitting channels may be attributed to the onset of ICF. Similar analysis of EFs using statistical model code PACE4 has been presented elsewhere [11]. 
In order to understand the onset of ICF in ${ }^{12} \mathrm{C}+{ }^{169} \mathrm{Tm}$ system, an attempt has been made to account for ICF contribution from experimentally measured EFs. Although, it is not possible to directly obtain the relative contribution of $\mathrm{CF}$ and ICF from the measurement of EFs, therefore some systematics has been followed [7]. As already mentioned, the enhancement in the experimentally measured production cross-sections over theoretical model predictions based on CF calculations may be attributed to the contribution from ICF. As suggested by Gomes et al.[7], the ICF contribution for individual channels has been deduced by subtracting CF cross-sections $\left(\sigma_{C F}\right)$ (predicted by theoretical model code) from the experimentally measured cross-sections $\left(\sigma_{E X P}\right)$ at respective projectile energies. The ICF fraction $\left(\sigma_{I C F}\right)$ for presently measured evaporation residues are plotted in Fig.2(left) as a function of projectile energy. Lines are drawn to guide the eyes.

In order to show how does ICF contribute to the total fusion cross-section $\left(\sigma_{T F}=\Sigma \sigma_{C F}+\Sigma \sigma_{I C F}\right)$, the overall ICF cross-section $\left(\Sigma \sigma_{I C F}\right)$ is plotted with the sum of all CF-channels $\left(\Sigma \sigma_{C F}\right)$ and $\sigma_{T F}$ in Fig.2(right). For an easy visualisation of increasing ICF contribution with energy, the value of $\Sigma \sigma_{C F}$ and $\sigma_{T F}$ are plotted on linear scale in this figure. As shown in this figure, the increasing separation between $\Sigma \sigma_{C F}$ and $\sigma_{T F}$ with projectile energy indicates energy dependence of ICF strength. For better insight into the projectile energy effect on ICF strength, the percentage fraction of ICF $\left(\mathrm{F}_{I C F}\right)$ in ${ }^{12} \mathrm{C}+{ }^{169} \mathrm{Tm}$ system has been deduced and plotted as a function of projectile energy in Fig.3(a), i.e., termed as ICF strength function. The ICF strength function defines empirical probability of ICF at different projectile energies. As shown in this figure, the value of $\mathrm{F}_{I C F}$ is found to be $\approx 7 \%$ at $\approx 59$ $\mathrm{MeV}$, i.e., $1.075 \mathrm{Vb}$ (only $7.5 \%$ above the barrier), and increases smoothly upto $\approx 18 \%$ at highest measured energy i.e., $1.64 \mathrm{Vb}$. It may be because of the fact that the projectile break-up probability of incident ion in the field of the target nucleus significantly increases with incident energy. This unexpected existence of ICF at such a low energy has been justified as a consequence of high input angular momenta imparted into the system due to the noncentral interactions $[3,4,17,18]$.

\subsection{Pro-Mass systematics}

The experiments presented in this paper have been performed to understand recently observed contradiction on Morgenstern's mass-asymmetry systematics [28]. Therefore, the values of $\mathrm{F}_{I C F}$ obtained in ${ }^{12} \mathrm{C}+{ }^{169} \mathrm{Tm}$ (from this work) and ${ }^{16} \mathrm{O}+{ }^{169} \mathrm{Tm}$ (from ref.[11]) systems are plotted as a function of $v_{r e l}$ in Fig.3(b). The energy axis is normalised to incorporate the Coulomb barriers of two systems compared in the figure, and to keep same observable as has been used in most of the previous studies. According to Morgenstern's systematics [28], ICF contributes significantly above $v_{\text {rel }} \approx 0.06$ (i.e., $6 \%$ speed of light). In this work, the values of $v_{r e l}$ are found to be in the range from $\approx 0.027$ (2.7\% of c) to $\approx 0.084(8.4 \%$ of $\mathrm{c})$ for ${ }^{12} \mathrm{C}$, and from $\approx 0.014$ ( $1.4 \%$ of $\mathrm{c})$ to $\approx 0.053(5.3 \%$ of $\mathrm{c}$ ) for
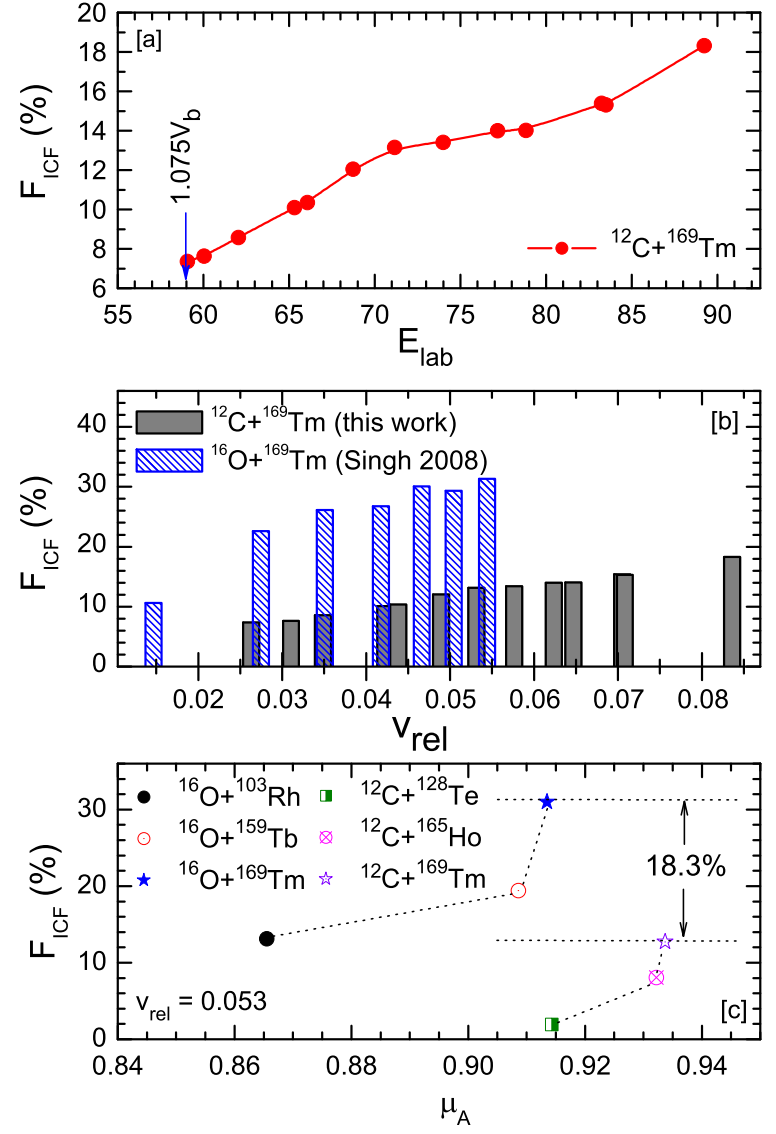

Figure 3. (a) the ICF strength function deduced from the analysis of experimental EFs of evaporation residues populated via $\mathrm{CF}$ and/or ICF in ${ }^{12} \mathrm{C}+{ }^{169} \mathrm{Tm}$ system (see text for description), (b) the value of $\mathrm{F}_{I C F}$ as a function of relative velocity $\left(\mathrm{v}_{\text {rel }}\right.$ ) for ${ }^{12} \mathrm{C},{ }^{16} \mathrm{O}+{ }^{169} \mathrm{Tm}$ (Singh 2008:[11]) systems, and (c) the values of $\mathrm{F}_{I C F}$ obtained for ${ }^{12} \mathrm{C}+{ }^{128} \mathrm{Te},{ }^{165} \mathrm{Ho},{ }^{169} \mathrm{Tm}$ and ${ }^{16} \mathrm{O}+{ }^{103} \mathrm{Rh},{ }^{159} \mathrm{~Tb},{ }^{169} \mathrm{Tm}$ systems as a function of mass asymmetry of interacting partners $\left(\mu_{A}\right)$ at a constant relative velocity (i.e., $\left.\mathrm{v}_{r e l}=0.053\right)$. The dotted lines drawn through the data points guide the eyes for individual $\left({ }^{12} \mathrm{C}\right.$ and $\left.{ }^{16} \mathrm{O}\right)$ projectiles.

${ }^{16} \mathrm{O}$. As per Morgenstern's mass-asymmetry systematics, no significant ICF contribution is expected at the given values of $v_{\text {rel }}$. However, the results presented in Fig.3(b) clearly demonstrate the onset of ICF at relatively lower value of $v_{\text {rel }}$ i.e, $\approx 0.027\left(\mathrm{~F}_{I C F} \approx 7 \%\right)$ in ${ }^{12} \mathrm{C}+{ }^{169} \mathrm{Tm}$ system, and at $\approx 0.014\left(\mathrm{~F}_{I C F} \approx 10 \%\right)$ in ${ }^{16} \mathrm{O}+{ }^{169} \mathrm{Tm}$ system. In both cases, the observed value of $\mathrm{F}_{I C F}$ is significant at well below the proposed onset value of $v_{\text {rel }}$ (i.e., $6 \%$ of c). Therefore, it can be inferred that the ICF starts competing with CF even at slightly above barrier energies.

Further, as can be noticed in Fig.3(b), the value of $\mathrm{F}_{\text {ICF }}$ for ${ }^{12} \mathrm{C}$-projectile is lower than that observed for ${ }^{16} \mathrm{O}$ projectile for the entire measured energy range. The difference in $\mathrm{F}_{I C F}$ for two systems $\left({ }^{12} \mathrm{C},{ }^{16} \mathrm{O}+{ }^{169} \mathrm{Tm}\right)$ can be seen clearly, which indicates the dependence of incomplete fusion fraction $\left(\mathrm{F}_{I C F}\right)$ on projectile type and/or on 
mass asymmetry of interacting partners $\left(\mu_{A}\right)$. The fact that both ${ }^{12} \mathrm{C}$ and ${ }^{16} \mathrm{O}$ are $\alpha$-cluster nuclei, which may break up into several combinations of $\alpha$-clusters. The probability of breakup increases with input $\ell$-values imparted into system due to the peripheral interactions [4]. Some of the breakup combinations which have been observed in previous studies are; $(a){ }^{12} \mathrm{C} \rightarrow{ }^{8} \mathrm{Be}+{ }^{4} \mathrm{He}(\alpha)$ and/or three $\alpha$ fragments, and $(b){ }^{16} \mathrm{O} \rightarrow{ }^{12} \mathrm{C}+{ }^{4} \mathrm{He},{ }^{8} \mathrm{Be}+{ }^{8} \mathrm{Be}$ and/or four $\alpha$ fragments. One or a group of fragments (in direct or successive mode) may fuse with target nucleus to form a partially fused composite system. The overlap between the interacting nuclei and the transformed mass depends on the number of nucleons occupying the overlapping volume. Therefore, ${ }^{16} \mathrm{O}$ may open up more ICF-channels as compared to ${ }^{12} \mathrm{C}$ induced reactions.

In order to refine projectile dependence and/or mass-asymmetry effect on $\mathrm{F}_{I C F}$, the values of $\mathrm{F}_{I C F}$ for nearby systems $\left({ }^{12} \mathrm{C}+{ }^{128} \mathrm{Te},{ }^{165} \mathrm{Ho},{ }^{169} \mathrm{Tm}\right.$ and $\left.{ }^{16} \mathrm{O}+{ }^{103} \mathrm{Rh},{ }^{159} \mathrm{~Tb},{ }^{169} \mathrm{Tm}\right)$ are deduced from the reanalysis of their EFs, and are plotted as a function of $\mu_{A}$ in Fig.3(c) at a constant value of $\mathrm{v}_{\text {rel }}=0.053$ (i.e., 5.3\% of speed of light). As demonstrated in this figure, the Morgenstern's mass-asymmetry systematics does not explain the variation of $\mathrm{F}_{I C F}$ with $\mu_{A}$ for given systems. However, the value of $\mathrm{F}_{I C F}$ increases with $\mu_{A}$ for individual projectiles (i.e., for ${ }^{16} \mathrm{O}$ and ${ }^{12} \mathrm{C}$ separately). It is interesting to note that the ${ }^{12} \mathrm{C}+{ }^{169} \mathrm{Tm}$ is a more mass-asymmetric $\left(\mu_{A}\right.$ $=0.9337)$ system than ${ }^{16} \mathrm{O}+{ }^{169} \mathrm{Tm}$ system $\left(\mu_{A}=0.9135\right)$, but the value of $\mathrm{F}_{I C F}$ is $18.3 \%$ higher than that observed for ${ }^{12} \mathrm{C}+{ }^{169} \mathrm{Tm}$ system. The aforementioned observations based on six projectile-target combinations strongly contradict Morgenstern's mass-asymmetry systematics and may be assumed as a supplement. The reconciliation of mass-asymmetry systematics achieved from these results may be termed as projectile-dependence-mass-asymmetry systematics, the Pro-Mass systematics.

\section{Summary and Conclusions}

This paper reports an insight into the projectile energy and mass-asymmetry $\left(\mu_{A}\right)$ dependence on the onset and strength of ICF. Existence of ICF at low incident projectile energy (i.e, at $7.5 \%$ above the barrier) is conclusively demonstrated. It has been found that the value of $\mathrm{F}_{I C F}$ increases from $\approx 7 \%$ to $\approx 18 \%$ with in the studied energy range (i.e., $1.02 \mathrm{~V}_{b}$ to $1.64 \mathrm{~V}_{b}$ ). A comparison of $\mathrm{F}_{I C F}$ for ${ }^{12} \mathrm{C}+{ }^{169} \mathrm{Tm}\left(\mu_{A}=0.9337\right)$ and ${ }^{16} \mathrm{O}+{ }^{169} \mathrm{Tm}$ $\left(\mu_{A}=0.9135\right)$ systems displays substantially higher ICF probability for ${ }^{16} \mathrm{O}+{ }^{169} \mathrm{Tm}$ system, though its less massasymmetric than ${ }^{12} \mathrm{C}+{ }^{169} \mathrm{Tm}$ system. The Morgenstern's mass-asymmetry systematics does not explain low energy ICF data of six nearby systems $\left({ }^{12} \mathrm{C}+{ }^{128} \mathrm{Te},{ }^{165} \mathrm{Ho},{ }^{169} \mathrm{Tm}\right.$ and ${ }^{16} \mathrm{O}+{ }^{103} \mathrm{Rh},{ }^{159} \mathrm{~Tb},{ }^{169} \mathrm{Tm}$ ) as a whole. However, the value of $\mathrm{F}_{I C F}$ is found to be in good agreement with $\mu_{A}$ separately for ${ }^{16} \mathrm{O}$ and ${ }^{12} \mathrm{C}$ projectiles, and increases with $\mu_{A}$ for individual projectiles . For the same target nuclei (i.e., ${ }^{169} \mathrm{Tm}$ ), the value of $\mathrm{F}_{I C F}$ for ${ }^{16} \mathrm{O}$ induced reaction is found to be $18.3 \%$ higher than that observed for ${ }^{12} \mathrm{C}$ induced reactions. As such, it can be inferred that the mass asymmetry systematics is valid only for individual projectile(s). The results presented in this paper supplements Morgenstern's mass asymmetry systematics, and the presentation of results in this particular fashion is termed as Pro-Mass systematics. The extension of this work using ${ }^{13} \mathrm{C},{ }^{14} \mathrm{~N}$ and ${ }^{18} \mathrm{O}$ beams with ${ }^{169} \mathrm{Tm}$ target would be interesting, and will be helpful for further refinement of present systematics.

Authors are thankful to the Director of IUAC New Delhi for extending all the facilities to perform these experiments. One of the authors PPS thanks to Prof. P. K. Raina (Dean A\&R) and Prof. M. K. Surappa (Director, IIT Ropar) for their kind support and motivation. BPS, RP and DPS thank to DST and UGC for financial support. AY thanks the SERB-DST for providing research funds through Young Scientist Scheme (Project No. SB/FTP/PS194/2013) under startup research grant.

\section{References}

[1] Alexis Diaz-Torres, J. Phys. G: Nucl. Part. Phys. 37, 075109 (2010).

[2] D. J. Hinde and M. Dasgupta, Phys. Rev. C 81, 064611 (2010).

[3] Pushpendra P. Singh et al., Phys. Rev. C 80, 064603 (2009).

[4] Pushpendra P. Singh et al., Phys. Lett. B 671, 20 (2009); Phys. Rev. C 78, 017602 (2008).

[5] L. R. Gasques et al., Phys. Rev. C 79, 034605 (2009); Phys. Rev. C74, 064615 (2006).

[6] Devendra P. Singh et al., Phys. Rev. C 81, 054607 (2010).

[7] P. R. S. Gomes et al., Phys. Lett. B 601, 20 (2004); Phys. Rev. C 73, 064606 (2006); Phys. Rev. Lett. B53, 1630 (1984).

[8] A. Diaz-Torres et al., Phys. Rev. Lett. 98, 152701 (2007).

[9] E. Z. Buthelezi et al., Nucl. Phys. A 734, 553 (2004).

[10] Unnati Gupta et al., Phys. Rev. C 80, 024613 (2009).

[11] Pushpendra P. Singh et al., Phys. Rev. C 77, 014607 (2008); Euro. Phys. Jour. A 34, 29 (2007) .

[12] Unnati Gupta et al., Nucl. Phys. A 811, 77 (2008).

[13] H. C. Britt and A. R. Quinton, Phys. Rev. 124, 877 (1961).

[14] J. Galin et al., Phys. Rev. C 9, 1126 (1974).

[15] Claudie Gerschel, Nucl. Phys. A 387, 297 (1982) .

[16] P. E. Hodgson, E. Gadioli, and E. Gadioli Erba, Introductory Nuclear Physics (Clarendon Press, Oxford, 1997), Chap. 23.

[17] J.Wilczyǹski et al., Phys. Rev. Lett. 45, 606 (1980); Nucl. Phys. A 373, 109 (1982).

[18] K. Siwek-Wilczyǹska et al., Phys. Rev. Lett. 42, 1599 (1979).

[19] J. H. Barker et al., Phys. Rev. Lett. 45, 424 (1980).

[20] D. J. Parker, J. Asher, T. W. Conlon, and I. Naqib, Phys. Rev. C 30, 143 (1984).

[21] T. Udagawa and T. Tamura, Phys. Rev. Lett. 45, 1311 (1980). 
[22] Pushpendra P. Singh et al., Eur. Phys. J. A 34, 29 (2007).

[23] J. R. Wu and I. Y. Lee, Phys. Rev. Lett. 45, 8 (1980).

[24] J. R. Wu, C. C. Chang, and H. D. Holmgren, Phys. Rev. Lett. 40, 1013 (1978); Phys. Rev. C19, 370 (1979).

[25] R. L. Robinson et al., Phys. Rev. C24, 2084 (1981).

[26] H. Utsunomiya et al., Phys. Lett. B 105, 135 (1981).

[27] I. Tserruya et al., Phys. Rev. Lett. 60, 14 (1988).

[28] H. Morgenstern et al., Z. Phys. A313, 39 (1983); Phys. Rev. Lett. 52, 1104 (1984); Z. Phys. A324, 443
(1986).

[29] S. Chakrabarty et al., Nucl. Phys. A678, 355-366 (2000).

[30] M. K. Sharma et al., Eur. Phys. Jour. A 31, 43-51 (2007); J. Phys. Soc. Jpn. 72, 1917 (2003).

[31] S. Gupta et al., Phys. Rev. C61, 064613 (2000).

[32] http://lise.nscl.msu.edu/pace4.html

[33] R. Bass; Nucl. Phys. A 231, 45 (1974).

[34] P. M. Endt, At. Data Nucl. Data Tables 26, 47 (1981).

[35] A. Gavron, Phys. Rev. C 21, 230 (1980). 